\title{
Pancreatic and Peripancreatic Tuberculosis
}

\author{
Eman Rashid Musa ${ }^{1}$ and Sushila B Ladumor ${ }^{2 *}$ \\ ${ }^{1}$ Clinical Fellow in Clinical Imaging Department, Body Imaging, Hamad Medical Corporation, Hamad General Hospital, Qatar
}

${ }^{2}$ Consultant Radiologist, Clinical Imaging Department, Hamad Medical Corporation, HGH, Doha, Qatar, Assistant Professor in Clinical Radiology, Weil Cornel Medical College, Qatar (WCMC-Q)

Submission: October 04, 2017; Published: October 16, 2017

*Corresponding author: Sushila Ladumor B, Consultant Radiologist, Clinical Imaging Department, Hamad Medical Corporation, HGH, Doha, Qatar, Assistant Professor in Clinical Radiology, Weil Cornel Medical College, Doha, Qatar (WCMC-Q), E-mail: drsbladumor@yahoo.com

\begin{abstract}
Pancreatic tuberculosis is a rare disease of the pancreas. Its clinical and imaging findings usually mimic pancreatic malignancy and preoperative diagnosis require a high index of clinical suspicion, particularly in immune compromised patients and immigrants from endemic areas of tuberculosis. In this article we present a case of isolated pancreatic and peripancreatictuberculosis simulating a pancreatic neoplasm.

Abbreviations: TB: Tuberculosis; PT: Pancreatic Tuberculosis; PN: Pancreatic Neoplasm; EUS: Endoscopic Ultra Sonography; MRI: Magnetic Resonance Imaging; MRCP: Magnetic Resonance Cholangio Pancreatography; CBD: Common Bile Duct; OB: Obstruction; AIDS: Acquired Immunodeficiency Syndrome
\end{abstract}

\section{Case Report}

\section{Clinical History}

A 24- year-old Nepalese man was presented to the emergency unit with history of right upper abdominal pain for one week duration. The pain is periodic and not shifting or radiating and associated with loss of appetite, fever and dark urine. He is a construction worker with no previous medical or surgical history and his last travel to his home country, Nepal, was 5 months back (Figures 1-3).

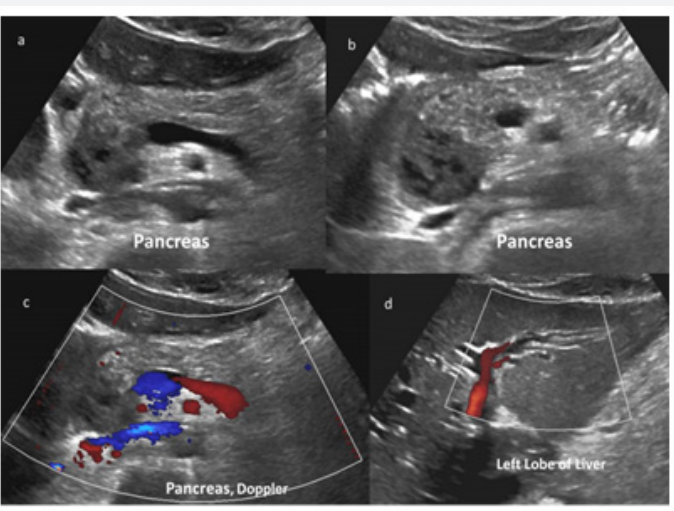

Figure 1: Ultrasound of upper abdomen with color Doppler (a, b, c \& d): The scan showed dilation of intra hepatic ducts (IHD) and the common bile duct (CBD) with $29 \times 27 \mathrm{~mm}$ hypo echoic mass noted at region of the pancreatic head. Pancreatic duct is not dilated. No other per pancreatic collection or ascites in upper abdomen. Portal and splenic vein are patent.

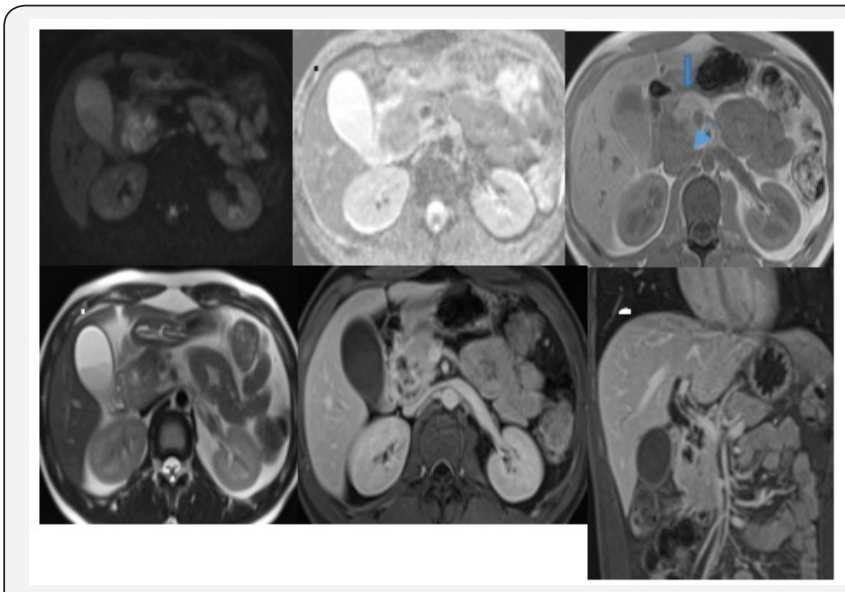

Figure 2: MRI: Reveals restriction of diffusion (a) which is relatively low in corresponding ADC (b). In-phase dual echo sequence (c) demonstrates low signal mass around pancreatic head (shown by thin arrow in image $\# \mathrm{c}$ ) with maintained T1 high signal in rest of pancreas (shown by thick arrow in image \#c), this focal lesion shows intermediated high signal in T2 weighted image (d). Post-contrast axial (e) and coronal (f) images demonstrate early and delayed persistent pattern of enhancement with multiple non-enhancing central areas impressive of cystic/necrotic changes. Distal CBD appears encased by focal mass with proximal dilatation. Rest of pancreas shows normal homogenous enhancement. 


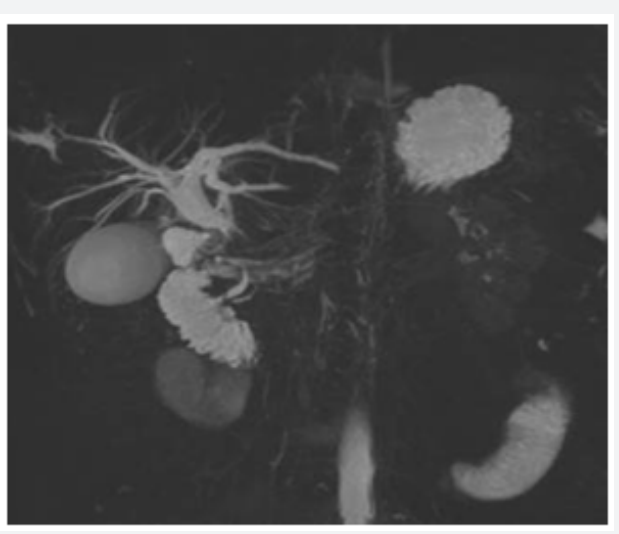

Figure 3: Magnetic resonance cholangiopancreatography (MRCP) showed $23 \mathrm{~mm}$ lengthen smooth stricture of mid and distal CBD and an upstream dilation of the intra and extra hepatic bile ducts. The pancreatic duct was normal in caliber.

\section{Patient's Blood Work-up}

The patient's initial laboratory analysis revealed a leukocytosis $(11.6 \times 103 / \mathrm{uL})$ and demonstrated cholestasis by increased serum levels of alkaline phosphates $(175 \mathrm{U} / \mathrm{L}$; normal range $4 \mathrm{U} / \mathrm{L}$ to $150 \mathrm{U} / \mathrm{L})$ and total bilirubin $(80.4 \mu \mathrm{mol} / \mathrm{L}$; normal range $3.4 \mu \mathrm{mol} / \mathrm{L}$ to $20.5 \mu \mathrm{mol} / \mathrm{L}$ ), with a direct bilirubin level of $62.3 \mu \mathrm{mol} / \mathrm{L}$ (normal range $0 \mu \mathrm{mol} / \mathrm{L}$ to $8.6 \mu \mathrm{mol} / \mathrm{L}$ ). C-reactive protein level was high measuring $29 \mathrm{mg} / \mathrm{L}$ (normal $<5 \mathrm{mg} / \mathrm{L}$ ). Tests for HIV and hepatitis B and C were negative. Patient was diagnosed as a case of obstructive jaundice and ultrasound examination was requested as first tool of investigation. The scan showed dilation of intra hepatic ducts (IHD) and the common bile duct (CBD) with $29 \times 27 \mathrm{~mm}$ hypo echoic mass noted at region of the pancreatic head. His chest radiograph was normal. Endoscopic Ultrasound (EUS) revealed hypo echoic mass in relation to the head of pancreas and peripancreatic region with CBD involvement. FNA-biopsy was performed with 21gauge needle and sent for histopathological evaluation.

Magnetic resonance imaging (MRI) was requested for further characterization of the mass and revealed $3 \mathrm{~cm}$ lobulated mass at pancreatic head and pancreatic duodenal groove, seen to be abutting anterior aspect of portal vein and encasing the mid and distal part of CBD. The mass appeared slightly bright in T2-weighted images and showed restriction of diffusion. Post contrast images demonstrate early and delayed persistent pattern of enhancement with multiple non-enhancing central areas impressive of cystic/necrotic changes. Few enlarged porta hepatislymph nodes were noted and there was no liver lesion. Magnetic resonance cholangiopancreatography (MRCP) showed $23 \mathrm{~mm}$ lengthen smooth stricture of mid and distal CBD and an upstream dilation of the intra- and extra hepatic bile ducts. The pancreatic duct was normal in caliber. Rest of the pancreas demonstrates normal signal intensity and post-contrast enhancement.

The main differential consideration was conglomerated peri pancreatic necrotic lymph nodes, most likely of tuberculosis origin, extending/involving into the pancreatic head. However because of CBD involvement, other differential such extrahepatic cholangiocarcinoma and pancreatic neoplasm were considered. The EUS-guided biopsy revealed epithelioid cells in a background of necrotic cells and debris consistent with necrotizing granulomatous lymphadenitis. Anti-tuberculosis therapy was initiated with moxifloxacin, amikacin and ethambutol and the patient pain is improving. To relive the CBD obstruction, Endoscopic retrograde cholangiopancreatogram (ERCP) for biliary stenting was attempted twice but failed to access the CBD. The liver function test was deteriorating and urgent Percutaneous Tran hepatic Cholangiogram (PTC) was performed with successful placement of $8.5 \mathrm{Fr}$ internal/ external drainage catheters. The patient has been shifted to the communicable disease unite to be followed as case of abdominal tuberculosis.

\section{Background}

Pancreatic and peripancreatic tuberculosis is an extremely uncommon disease, presenting as hypo echoic mass on ultrasonography and imaging mimicking malignancy. Consequently, it represents a diagnostic challenge.

\section{Introduction}

Tuberculosis (TB) is a potentially systemic disease that can infect any organ and system. Abdominal infection with tuberculosis commonly affects the spleen, liver, and ileocecal region. Pancreatic tuberculosis is an extremely rare disease, especially when it is isolated in the pancreas. Pancreatic and peripancreatic tuberculosis (PPT) is a rare non-neoplastic lesion and its clinical and radiological findings may be similar to those of pancreatic malignancy [1]. Therefore, diagnosis of the PPT is very difficult, and most of previously reported cases were diagnosed after exploratory laparotomy for suspected pancreatic malignancy. Pancreatic and peripancreatic TB presents with a wide spectrum of symptoms such as abdominal pain, constitutional symptoms, obstructive jaundice, iron-deficiency anemia, pancreatic abscess, massive gastro-intestinal bleeding, acute/chronic pancreatitis, secondary diabetes, splenic vein thrombosis and a pancreatic mass mimicking malignancy. The frequency of PPT cases in developing countries has increased in recent years.

\section{Discussion}

Tuberculosis (TB) is a life threating disease that affects any system organ and its prevalence has been raised due to the acquired immunodeficiency syndrome (AIDS) outbreak and the increasing number of drug-resistant strains of Mycobacterium tuberculosis [2]. Tuberculosis is also known to be associated with poverty, deprivation and immigrants from Third-World countries. The primary site of TB is usually lung and abdominal involvement occurs in about $11 \%$ of patients with extrapulmonary tuberculosis. The common site of abdominal tuberculosis is the gastrointestinal tract (especially the ileocecal 
junction), peritoneum, lymph nodes and solid viscera. Clinical presentation of abdominal TB depend on the site of involvement and tuberculosis at unusual locations can mimic most common disease of that organ, e.g. pancreas- carcinoma, pancreatitis, and abscess [3].

Although imaging findings could raise the possibility of tuberculosis, they are neither non-specific nor path gnomonic for pancreatic TB. Pancreatic TB usually appears in imaging as hypo echoic/low-attenuation mass, most frequently in the head of the pancreas, or diffuse enlargement of the pancreas [4]. Per pancreatic and peri portal lymphadenopathy with peripheral ring enhancement, ascites and mural thickening affecting the ileocecal region, are ancillary findings that support the diagnosis. The common bile duct (CBD) and the pancreatic duct are usually normal, even if the mass is localized centrally in the head of the pancreas [5]. However in our case the proximal CBD were encased by the mass and associated upstream dilatation of IHD and CBD were present. Histological result was necessary for establishing the diagnosis of pancreatic TB. Most of the patients with pancreatic TB respond well to anti-tuberculous therapy treated over a period of 6-12 months. However for those patients with obstruction due to larger mass, minimally invasive procedures such as endoscopic internal drainage and percutaneous catheter drainage or biliary stenting, should be considered [6].

\section{Conclusion}

Pancreatic tuberculosis is a challenging diagnosis due rarity of the disease and its insidious presentations. It should be considered in the differential diagnosis of focal pancreatic mass in patients presented with obstructive jaundice. However, if doctors are aware of its clinical features and conduct appropriate investigations with multiple modalities including US, MRI, CT scan and ultrasound-guided FNA or laparoscopic biopsy, diagnosis of pancreatic tuberculosis without laparotomy is possible and the disease can be effectively treated with anti tuberculosis drugs.

\section{References}

1. Feng Xia, Ronnie Tung Ping Poon, Shu Guang Wang, Ping Bie, Xue Quan Huang, et al. (2003) Tuberculosis of pancreas and peripancreatic lymph nodes in immune competent patients: experience from China. World ] Gastroenterol 9(6): 1361-1364.

2. Katherine JTo'o, BS Steven S Raman, Nam C Yu, Young Jun Kim, Tyler Crawford, et al. (2005) Pancreatic and Peripancreatic Diseases Mimicking Primary Pancreatic Neoplasia. Radio Graphics 25(4).

3. Davis SD, Yankelevitz DF, Williams T (1993) Pulmonary tuberculosis in immune compromised hosts: epidemiological clinical and radiological assessment. Semin Roentgenol 28:119-130.

4. Takhtani D, Gupta S, Suman K, Kakkar N, Challa S, et al. (1996) Radiology of pancreatic tuberculosis: A report of three cases. Am J Gastroenterol 91(9): 1832-1834.

5. De Backer AI, Mortelé KJ, Bomans P, De Keulenaer BL, Vanschoubroeck IJ, et al. (2005) Tuberculosis of the pancreas: MRI features. AJR Am J Roentgenol 184(1): 50-54.

6. Meesiri S (2012) Pancreatic tuberculosis with acquired immunodeficiency syndrome: a case report and systematic review. World J Gastroenterol 18(7): 720-726.

\section{Your next submission with Juniper Publishers} will reach you the below assets

- Quality Editorial service

- Swift Peer Review

- Reprints availability

- E-prints Service

- Manuscript Podcast for convenient understanding

- Global attainment for your research

- Manuscript accessibility in different formats

( Pdf, E-pub, Full Text, Audio)

- Unceasing customer service

Track the below URL for one-step submission https://juniperpublishers.com/online-submission.php 